\title{
Determination of the total LHC Higgs boson Cross Section in Gluon Fusion
}

\section{Franz Herzog*}

Nikhef Theory Group, Science Park 105, 1098 XG Amsterdam, NL

E-mail: fherzog@nikhef.nl

We review the status of calculations for the inclusive Higgs boson cross section in the gluon fusion production channel. In particular we discuss the size of known QCD and electroweak corrections, their residual uncertainties as well as the size of other missing corrections.

38th International Conference on High Energy Physics 3-10 August 2016

Chicago, USA

${ }^{*}$ Speaker. 


\section{Introduction}

The discovery of the Higgs boson at the LHC $[1,2]$ represents one of the greatest achievements of particle physics. To measure the Higgs boson's properties and uncover its true nature will represent one of the main tasks of the future LHC program. While this requires further precise measurements from the experimental side it also requires at least equally precise theoretical predictions for coupling extractions to be meaningful and to make sense out of the data. Due to the slow perturbative convergence of the QCD corrections in the gluon fusion channel, the dominant Higgs boson production channel at the LHC in the Standard Model (SM), this has been a particularly challenging task. Determining the size of these QCD corrections required in particular the computation of the next-to-next-to-next-to-leading order (N3LO) QCD correction, which was provided about a year ago in [3]. By giving prescriptions for the estimation of residual uncertainties, a determination of the total Higgs Boson cross section and its uncertainty was provided in [4]. In the following we will review the calculations of QCD and electroweak correction and discuss the size of the missing higher order corrections.

\section{Higgs Boson Production in Gluon Fusion}

The Higgs boson cross section can be written as the following convolution integral,

$$
\sigma_{P P \rightarrow H+X}=\sum_{i j} \int d x_{1} d x_{2} f_{i}\left(x_{1}\right) f_{j}\left(x_{2}\right) \hat{\sigma}_{i j \rightarrow X H}\left(\hat{s},\left\{g_{s}, g_{W}, . .\right\},\left\{m_{H}, m_{t}, m_{b} . .\right\}\right),
$$

where $\hat{s}=x_{1} x_{2} S$ and $S$ is the collider energy. The sum runs over all partons $i$ in the proton, whose parton distribution function (PDF) is given by $f_{i}(x)$ for a given parton $i$ with longitudinal momentum fraction $x$. While the parton densities are essentially non-perturbative objects the partonic cross sections $\sigma_{i j \rightarrow P H}$ can be computed in a perturbative expansion around the various coupling constants, which we denoted by $\left\{g_{s}, g_{W} ..\right\}$. Besides coupling constants the partonic cross section further depends on the mass parameters of all the Standard Model particles, primarily the Higgs boson mass, $m_{H}$, but also for example on that of the top quark, $m_{t}$, the coupling to which is responsible for most of the gluon fusion cross section in the SM. Of particular interest are, besides the top production modes, electroweak corrections, mixed QCD-electroweak corrections and bottom quark mediated production modes. Beyond the next-to-leading order (NLO) the QCD corrections are known only in an effective theory which treats the top quark as infinitely heavy. In this heavy quark effective theory (HQET) the QCD corrections are now known to N3LO [3]. Electroweak corrections are known at NLO, and mixed QCD-electroweak corrections are known at NLO $[9,10,11,12]$. Sub-leading top quark mass effects are known at next-to-next-to-leading order (NNLO) [7, 8]. The various pieces can be combined using the following master formula:

$$
\hat{\sigma}_{i j \rightarrow X H} \simeq R_{L O}\left(\hat{\sigma}_{i j, E F T}+\delta_{t} \hat{\sigma}_{i j, E F T}^{N N L O}+\delta \hat{\sigma}_{i j, E W}\right)+\delta \hat{\sigma}_{i j, e x ; t, b, c}^{L O}+\delta \hat{\sigma}_{i j, e x ; t, b, c}^{N L O} .
$$

Let us briefly explain the different ingredients which enter this equation. Here $R_{L O}$ denotes the ratio of the leading order (LO) cross section mediated by the top quark divided by its large top quark mass limit. By rescaling the HQET result with this factor the validity of the effective theory is improved considerable. By $\hat{\sigma}_{i j, E F T}$ we denote the partonic cross section calculated in HQET, 


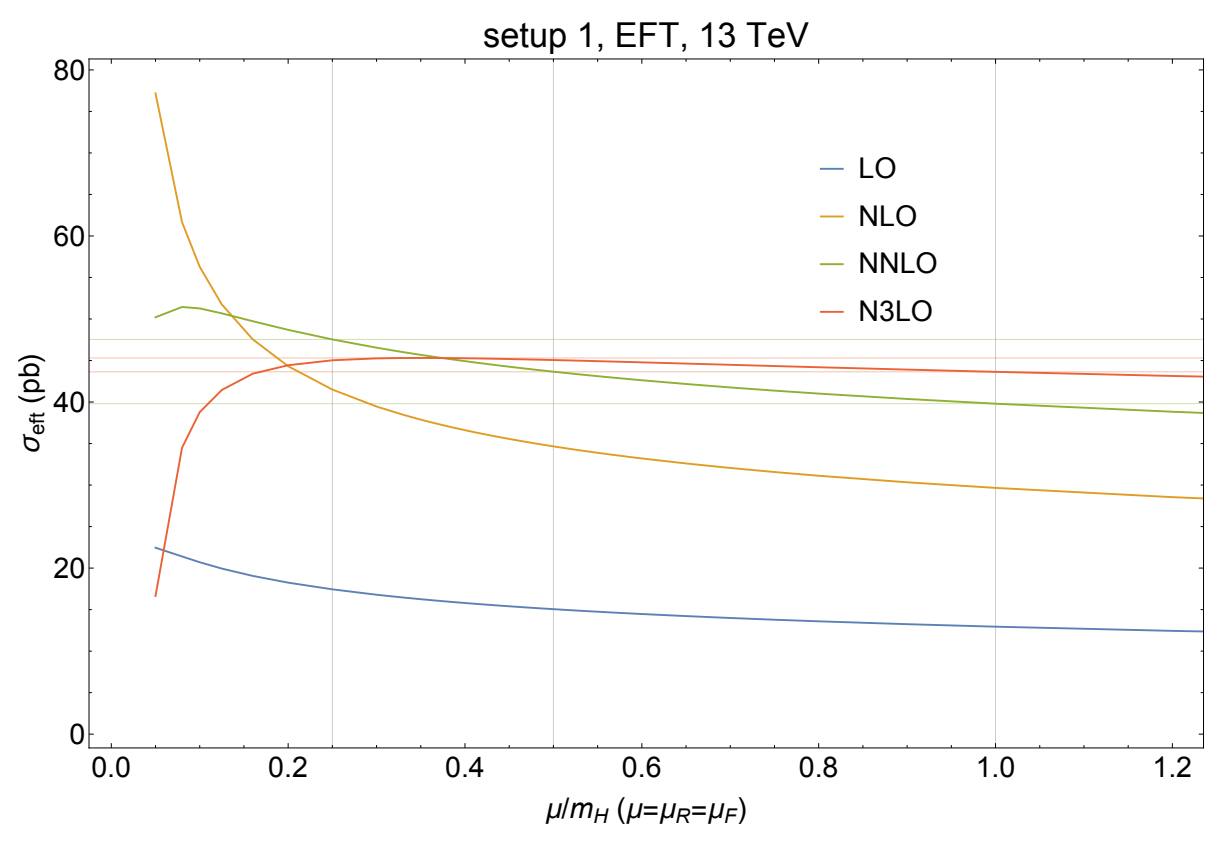

Figure 1: Simultaneous variation of both renormalisation and factorisation scales of the cross-section.

including QCD corrections up to N3LO and EW corrections up to NLO in the limit of large $W, Z$ mass. By $\delta_{t} \hat{\sigma}_{i j, E F T}^{N N L O}$ we denote sub-leading heavy top quark mass effects at NNLO in QCD. With $\delta \hat{\sigma}_{i j, E W}$ we denote a correction factor to include the full electroweak corrections at NLO. The terms $\delta \hat{\sigma}_{i j, e x ; t, b, c}^{L O}$ and $\delta \hat{\sigma}_{i j, e x ; t, b, c}^{N L O}$ allow us to include exact top and bottom quark mass effects at LO and NLO $[5,6]$.

\section{Sources of Uncertainty}

In the following we list different sources of uncertainty which enter the inclusive Higgs boson cross section in gluon fusion. We summarise in particular the prescriptions outlined in [4].

\section{- PDF Uncertainties}

These are estimated by the PDF provider. In [4] the PDF4LHC15 set [13] was used for the central predictions.

\section{- Perturbative Uncertainties}

Perturbative Uncertainties in QCD ( $\delta$ (scale) ) are estimated by a varying, up and down by a factor of two, renormalisation and factorisation scales around the central scale $\mu_{R}=\mu_{F}=$ $m_{H} / 2$. This scale variation is shown in Fig 1 . for the EFT. The factorisation scale dependence is milder than that of the renormalisation scale. The approach was validated by comparing it to threshold resumations using several different prescriptions. Uncertainities $(\delta(E W))$ stemming from unknown mixed QCD-electroweak effects were estimated by varying the size of the eletroweak Wilson coefficients. Missing top quark mass effects $\left(\delta\left(1 / m_{t}\right)\right)$ were estimated by studying the size of subleading mass corrections at NNLO. Missing bottom and charm 


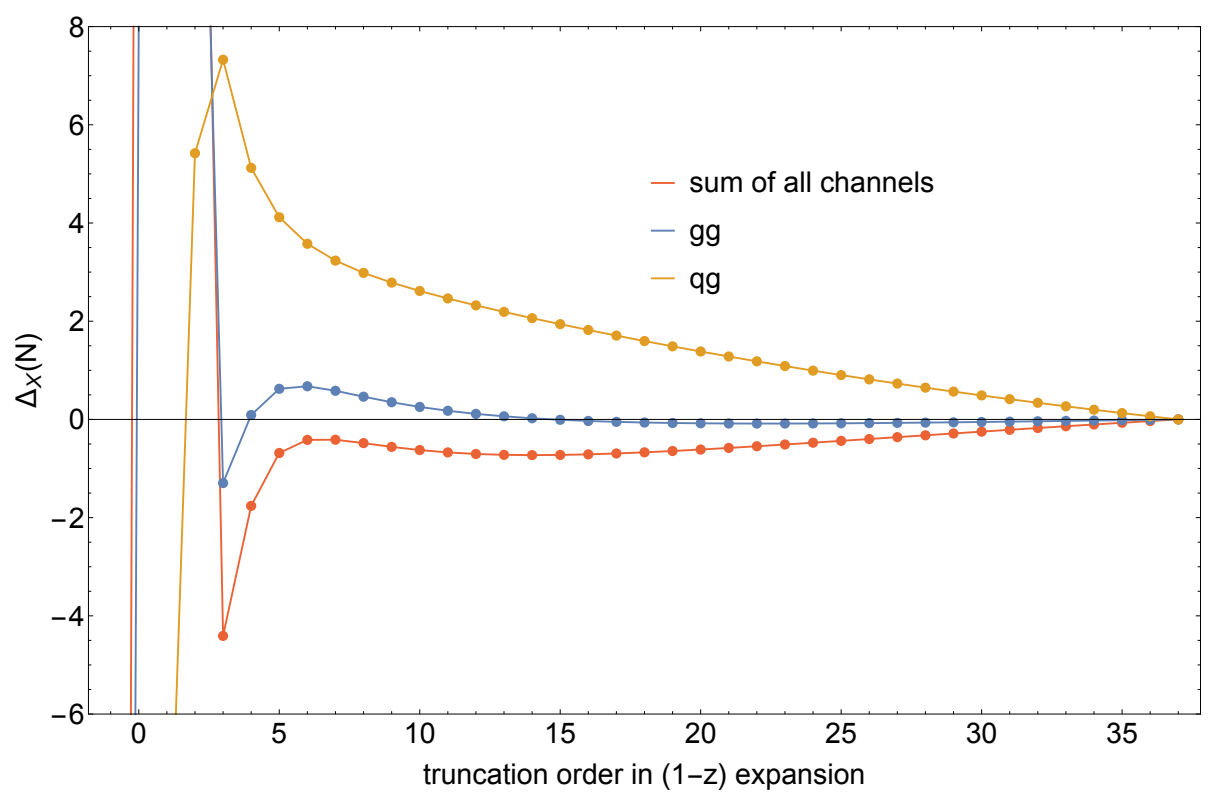

Figure 2: The plot shows the percent difference of the threshold expansion truncated at a given order to the result where all known orders are taken into account. The threshold expansion is an expansion around the limit $z=m_{H}^{2} / \hat{s}=1$

quark mass effects $(\delta(t, b, c))$ were estimated by assuming similar K-factors for their QCD corrections as are observed in the effective theory.

\section{- Parameteric Mass and Coupling Uncertainties}

The impact of the parameteric uncertainties of the strong coupling constant is included in the PDf uncertainty. The size of parametric mass parameter uncertainties was studied by considering experimental uncertainties for the top, bottom and charm masses. It turns out that these uncertainties are entirely negligible.

\section{- PDF Theory Uncertainty}

The PDF Theory Uncertainty ( $\delta($ PDF-TH $)$ ) is due to the lacking of N3LO PDFs, the extraction of which will be important in the future. The size of this uncertainty was estimated as the difference of computing the NNLO cross section with NLO or NNLO PDFs. The feature that N3LO PDFs are likely closer to the NNLO PDFs than NNLO PDFs are to those at NLO was incorporated by dividing with a factor of two.

\section{- Threshold Expansion Uncertainties}

Another source of uncertainty ( $\delta$ (trunc)) is related to a threshold expansion which was used in the computation of the N3LO QCD corrections. These uncertainties were estimated by studying the size of higher order terms. A plot showing these terms as a function of the truncation order is presented in Fig 2.

For a Higgs boson of mass $m_{H}=125 \mathrm{GeV}$ at the $13 \mathrm{TeV}$ LHC we find using the PDF4LHC15 
pdf set the following result:

$$
\sigma=48.58 \mathrm{pb}_{-3.27 \mathrm{pb}(-6.72 \%)}^{+2.22 \mathrm{pb}(+5 \%)} \text { (theory) } \pm 1.56 \mathrm{pb}(3.20 \%)\left(\mathrm{PDF}+\alpha_{s}\right) .
$$

The total cross section is broken down into its different components as follows:

$$
\begin{aligned}
& 48.58 \mathrm{pb}=16.00 \mathrm{pb} \quad(+32.9 \%) \quad(\mathrm{LO}, \mathrm{rEFT}) \\
& +20.84 \mathrm{pb} \quad(+42.9 \%) \quad(\mathrm{NLO}, \mathrm{rEFT})
\end{aligned}
$$

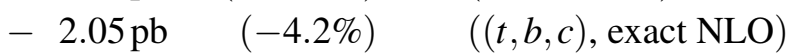

$$
\begin{aligned}
& +9.56 \mathrm{pb} \quad(+19.7 \%) \quad(\mathrm{NNLO}, \mathrm{rEFT}) \\
& +0.34 \mathrm{pb} \quad(+0.7 \%) \quad\left(\mathrm{NNLO}, 1 / m_{t}\right) \\
& +2.40 \mathrm{pb} \quad(+4.9 \%) \quad(\mathrm{EW}, \mathrm{QCD}-\mathrm{EW}) \\
& +1.49 \mathrm{pb} \quad(+3.1 \%) \quad\left(\mathrm{N}^{3} \mathrm{LO}, \mathrm{rEFT}\right)
\end{aligned}
$$

The breakdown of the theoretical uncertainty is summarised in the following table:

\begin{tabular}{cccccc}
\hline$\delta$ (scale $)$ & $\delta$ (trunc) & $\delta($ PDF-TH $)$ & $\delta($ EW $)$ & $\delta(t, b, c)$ & $\delta\left(1 / m_{t}\right)$ \\
\hline $\begin{array}{c}+0.10 \mathrm{pb} \\
-1.15 \mathrm{pb}\end{array}$ & $\pm 0.18 \mathrm{pb}$ & $\pm 0.56 \mathrm{pb}$ & $\pm 0.49 \mathrm{pb}$ & $\pm 0.40 \mathrm{pb}$ & $\pm 0.49 \mathrm{pb}$ \\
\hline${ }_{-2.37 \%}^{+0.21 \%}$ & $\pm 0.37 \%$ & $\pm 1.16 \%$ & $\pm 1 \%$ & $\pm 0.83 \%$ & $\pm 1 \%$ \\
\hline
\end{tabular}

To obtain the theory uncertainty in eq.(3.1) we combine all the uncertainties in the previous table linearly. While a complete $100 \%$ correlation of the uncertainties may appear overly conservative, however a certain degree of correlation among these uncertainties can not be disregarded and must be assumed to be present from the way in which each of them was estimated. For instance the uncertainty estimations for electroweak corrections and quark mass effects were based on QCD $\mathrm{K}$-factors which were found in the effective theories at NLO, NNLO and N3LO. This clearly correlates the uncertainties of electroweak corrections and quark mass effects with the scale uncertainties which make up the theory uncertainty of the effective theory K-factors.

\section{Conclusions}

A major reduction of the theoretical uncertainty of the LHC Higgs boson cross section was achieved through the inclusion of the N3LO QCD corrections. Although the remaining scale variation of the QCD corrections is now of the order 2-3\% a linear combination with other sources of uncertainty still results in a final theory uncertainty of about 4-6\%. To meet the precision goals of the LHC further efforts will be required in order to further pin down the individual sources of these uncertainties and thereby allow for their decorrelation.

\section{References}

[1] G. Aad et al. [ATLAS Collaboration], Observation of a new particle in the search for the Standard Model Higgs boson with the ATLAS detector at the LHC, Phys. Lett. B 716 (2012) 1, arXiv:1207.7214.

[2] S. Chatrchyan et al. [CMS Collaboration], Observation of a new boson at a mass of $125 \mathrm{GeV}$ with the CMS experiment at the LHC, Phys. Lett. B 716 (2012) 30, arXiv:1207.7235. 
[3] C. Anastasiou, C. Duhr, F. Dulat, F. Herzog and B. Mistlberger, Higgs Boson Gluon-Fusion Production in QCD at Three Loops," Phys. Rev. Lett. 114 (2015) 212001 arXiv:1503.06056 .

[4] C. Anastasiou, C. Duhr, F. Dulat, E. Furlan, T. Gehrmann, F. Herzog, A. Lazopoulos and B. Mistlberger, High precision determination of the gluon fusion Higgs boson cross-section at the LHC, JHEP 1605 (2016) 058, arXiv:1602.00695.

[5] M. Spira, A. Djouadi, D. Graudenz and P. M. Zerwas, Higgs boson production at the LHC, Nucl. Phys. B 453 (1995) 17, [hep-ph/9504378].

[6] R. Harlander and P. Kant, Higgs production and decay: Analytic results at next-to-leading order QCD, JHEP 0512 (2005) 015, [hep-ph/0509189].

[7] R. V. Harlander and K. J. Ozeren, Finite top mass effects for hadronic Higgs production at next-to-next-to-leading order, JHEP 0911 (2009) 088, arXiv:0909.3420.

[8] A. Pak, M. Rogal and M. Steinhauser, Finite top quark mass effects in NNLO Higgs boson production at LHC, JHEP 1002 (2010) 025 arXiv:0911.4662.

[9] U. Aglietti, R. Bonciani, G. Degrassi and A. Vicini, Two loop light fermion contribution to Higgs production and decays, Phys. Lett. B 595 (2004) 432, hep-ph/0404071.

[10] S. Actis, G. Passarino, C. Sturm and S. Uccirati, NLO Electroweak Corrections to Higgs Boson Production at Hadron Colliders, Phys. Lett. B 670 (2008) 12, arXiv:0809.1301.

[11] S. Actis, G. Passarino, C. Sturm and S. Uccirati, NNLO Computational Techniques: The Cases H $\rightarrow$ gamma gamma and $H \longrightarrow g$, Nucl. Phys. B 811 (2009) 182, arXiv:0809.3667.

[12] C. Anastasiou, R. Boughezal and F. Petriello, Mixed QCD-electroweak corrections to Higgs boson production in gluon fusion, JHEP 0904 (2009) 003 arXiv:0811.3458.

[13] J. Butterworth et al., PDF4LHC recommendations for LHC Run II, J. Phys. G 43 (2016) 023001, arXiv:1510.03865. 